\title{
Heartbeat: Chocolate and atrial fibrillation
}

Moderate chocolate consumption has been associated with lower rate of adverse cardiac events and mortality, with the mechanism of this association postulated to be the vasodilatory, antioxidant and anti-inflammatory effects of flavanols in chocolate. Motstofsky and colleagues ${ }^{1}$ hypothesized that the anti-inflammatory and anti-platelet effects of chocolate might also be associated with a lower rate of atrial fibrillation or flutter (AF). In the over 55 thousand subjects in the population based Danish Diet, Cancer and Health Study, there were 3346 cases of AF (6\%) over a median 13.5 years. This data showed that the rate of AF was lower for people consuming chocolate more than once a month, with a hazard ratio (HR) of 0.90 for $1-3$ servings/month, HR 0.83 for 1 serving/week, HR 0.80 for 2-6 servings per week and HR 0.84 for $\geq 1$ serving/day, with similar results in women and men. (figure 1) Although confounding effects are difficult to exclude with certainty, the authors conclude that this data suggests that moderate chocolate intake may be inversely associated with AF risk.

This is good news for chocolate lovers but is it too good to be true? Should we recommend our patients eat more chocolate or are there more, possibly more effective, approaches to reducing the incidence of AF? In the accompanying editorial, Pokorney and Piccini ${ }^{2}$ put this data in context by reminding us that us to $60 \%$ of AF cases can be attributed to modifiable risk factors, including hypertension, inactivity, obesity, diabetes and smoking (figure 2). In addition, subjects with higher chocolate intake tend to be healthier with less hypertension, less diabetes and higher levels of education. Even so, they conclude that "studies like the Danish chocolate study are important because we need to identify additional potential targets for AF prevention. Pragmatic randomised trials that focus on the efficacy of AF prevention strategies, as well as implementation trials that address dissemination, are needed. The highest quality care for $\mathrm{AF}$ is to prevent it in the first place. It will be interesting to see if chocolate is an effective preventive therapy.

Correspondence to Professor Catherine M Otto University of Washington, Division of Cardiology, Seattle, Washington, USA; cmotto@uw.edu, cmotto@u. washington.edu

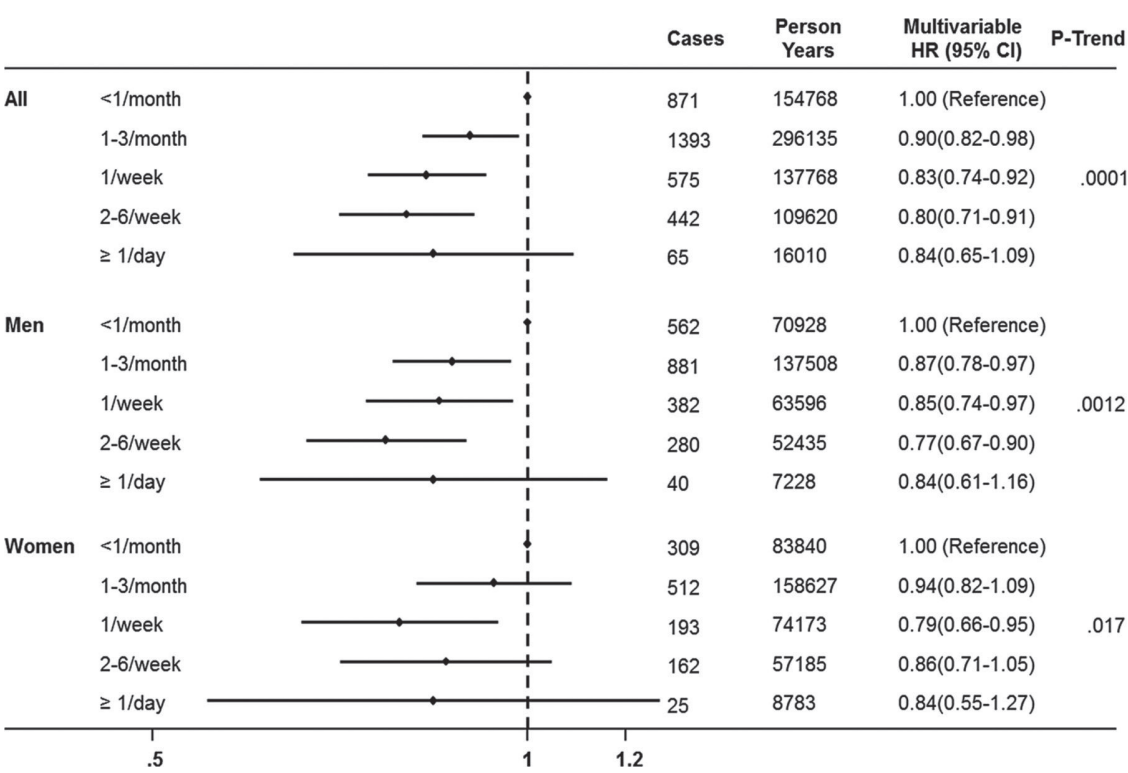

Figure 1 Multivariable hazard ratios (HRs) and 95\% confidence intervals (Cls) according to frequency of chocolate intake in the Danish Diet, Cancer, and Health Study. $p$ trend is the value for linear component of trend. Age was the time scale in the Cox models and we adjusted for total calories, sex, BMI, systolic blood pressure ( $\mathrm{mm} \mathrm{Hg}$ ), total serum cholesterol (continuous), coffee consumption (continuous), alcohol consumption (g/day), smoking status (never, former, current), years of education beyond elementary school $(0,<3,3-4,>4$ years), hypertension (yes/no), diabetes mellitus (yes/no) and cardiovascular disease (yes/no). ${ }^{1}$

Perhaps what is bad for the pancreas is good for the atria."

Another interesting paper in this issue addresses the clinical problem of predicting an individual patient's risk of bleeding with dual anti-platelet therapy (DAPT) after an

acute coronary syndrome (ACS). Based on outcomes in the 9240 patients in the TRIOLOGY ACS trial (Targeted Platelet Inhibition to Clarify the Optimal Strategy to Medically Manage ACS), Alfredsson and colleagues used the GUSTO (Global Use

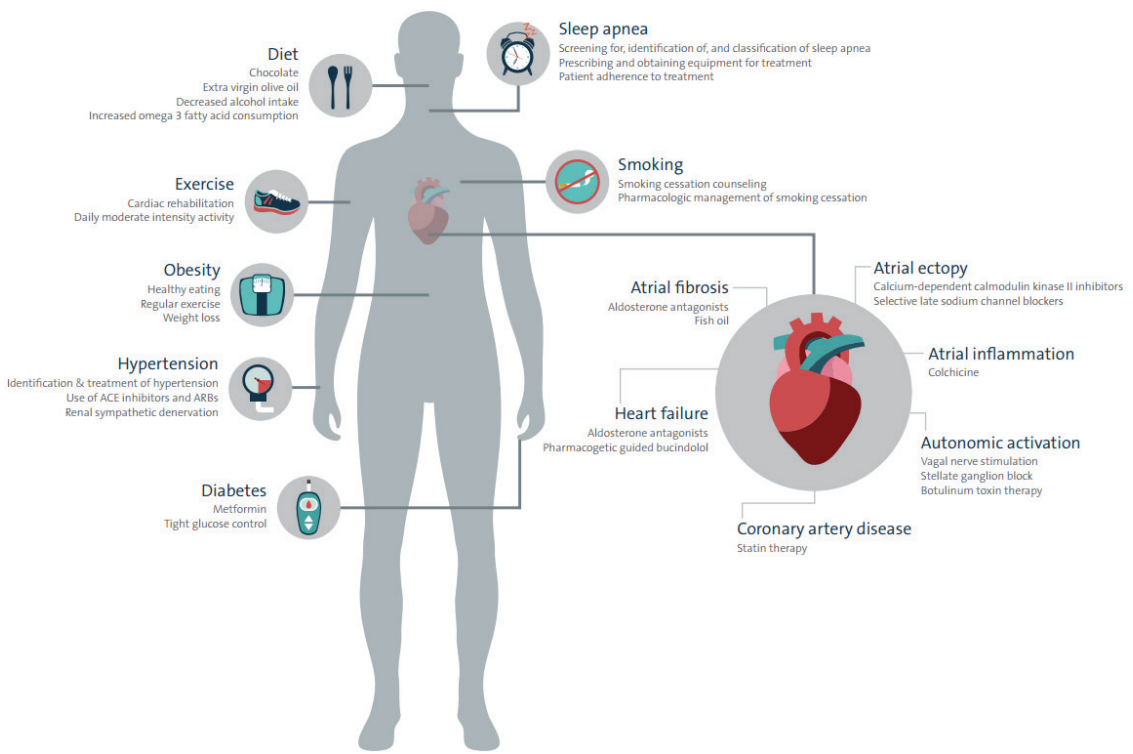

Figure 2 Targets for primary prevention of atrial fibrillation (AF) 2 . 


\section{CTA $50-70 \%$ LAD stenosis}

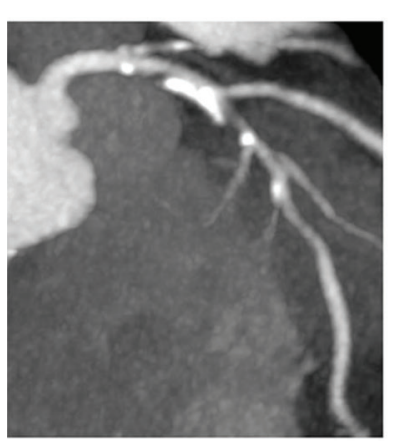

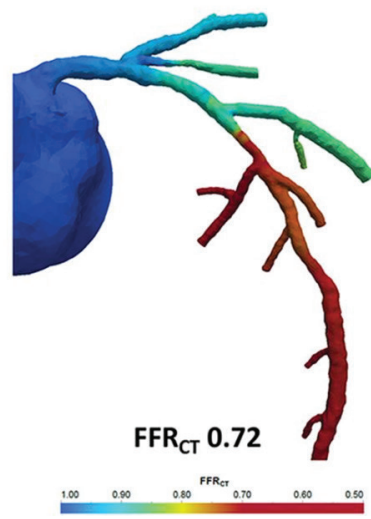

Angio $70 \%$ LAD stenosis

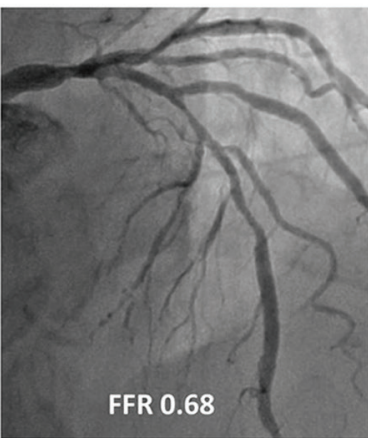

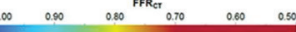

Figure 3 eFFRCTexample. A 72-year-old man with typical chest pain and cardiovascular risk factors for diabetes mellitus, hypertension, hyperlipidaemia and smoking underwent a coronary CTA. This showed a moderate calcified plaque (50\%-70\%) within the mid left anterior descending artery. The FFRCT value of the LAD was 0.72 , and the patient was subsequently listed for angiography and percutaneous coronary intervention. Invasive FFR was concordant with the FFRCT value and PCI was subsequently performed. CTA, computed tomographic angiography; FFR, fractional flow reserve; FFRCT, fractional flow reserve by coronary CTA; LAD, left anterior descending artery; $\mathrm{PCl}$, percutaneous coronary intervention.

of Strategies to Open Occluded Arteries) and TIMI (Thrombolysis In Myocardial Infarction) definitions of bleeding to assess the validity of a proposed risk score derived from 10 baseline predictors, including age, sex, creatinine, beta blocker use, hemoglobin, history of peptic ulcer disease, unstable angina versus myocardial infarction, whether angiography was performed, systolic blood pressure and weight (http:// trilogyacs-bleedingmodel-01.oit.duke.edu/).

Commenting on this proposed TRILOGY-ACS risk score for bleeding in patients on DAPT after an ACS, Wilson and Newby ${ }^{3}$ discuss the need to balance bleeding risk against the risk of thromboembolic events, as well as the challenges in using a complicated score that is not easily estimated at the bedside. They conclude: "Ideally, risk prediction tools are developed that allow modifiable longitudinal risk estimates to be generated at any given point, taking into account known dynamic variables and the time dependent severity of an event. Whether it is feasible to assimilate all

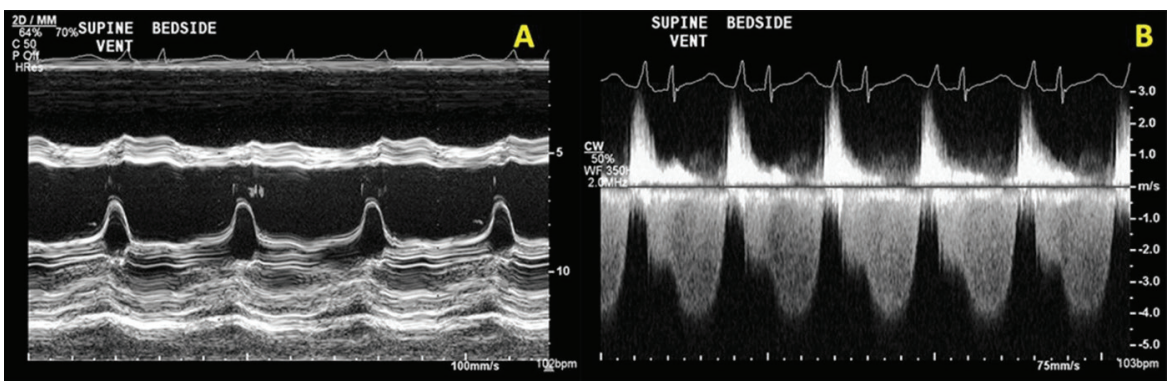

Figure 4 (A) M-mode recording of the mitral valve. (B) Continuous-wave Doppler of transmitral flow. ${ }^{8}$ these factors into one unifying and implementable model remains to bedetermined. Until then, the TRILOGY ACS bleeding risk models presented by Alfredsson and colleagues ${ }^{4}$ represent a significant step forward in predicting long-term bleeding risks with DAPT after ACS to facilitate clinical decision making on an individual level."

The choice of a bare metal (BMS) versus drug-eluting stent (DES) in patients with cardiogenic shock (CS) complicating an acute myocardial infarction remains controversial. In the randomised Intra-aortic Balloon Pump in Cardiogenic Shock II trial $(n=600)$ and the associated registry $(\mathrm{n}=183), 58 \%$ received a BMS with a risk-factor adjusted 1-year outcome similar to those treated with a DES. ${ }^{5}$ Küble and Reczuch $^{6}$ raise the concerns that CS may alter the pharmacodynamics of antiplatelet agents, therapeutic hypothermia may further impact drug efficacy, late stent thrombosis may be an issue, and details of stent implantation may also affect outcomes. They conclude: "there are still

more questions than answers regarding the issue of optimal stent for patients with AMI and CS." Even so, "probably prompt and durable revascularisation together with powerful intravenous antiplatelet agent and prompt use of mechanical circulatory support play much more important role than the kind of stent."

The Education in Heart article ${ }^{7}$ in this issue provides a primer on how to measure fractional flow reserve on coronary computed tomographic angiography, an approach that is likely to become an important clinical tool in the near future. (figure 3). One of the Image Challenge questions ${ }^{8}$ in this issue, asks you to choose the most appropriate definitive therapy for a 21-year-old women with a 2-month history of dyspnea on exertion, a physical exam showing heart failure with both a systolic and diastolic murmur and these echocardiographic findings (figure 4).

Competing interests None declared.

Provenance and peer review Commissioned; internally peer reviewed.

(c) Article author(s) (or their employer(s) unless otherwise stated in the text of the article) 2017. All rights reserved. No commercial use is permitted unless otherwise expressly granted.

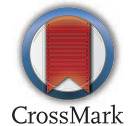

To cite Otto CM. Heart 2017:103:1139-1140.

Published Online First 22 June 2017

Heart 2017:103:1139-1140.

doi:10.1136/heartjnl-2017-312025

\section{REFERENCES}

1 Mostofsky E, Berg Johansen M, Tjønneland A, et al. Chocolate intake and risk of clinically apparent atrial fibrillation: the Danish Diet, Cancer, and Health Study. Heart 2017;103:1163-7.

2 Pokorney SD, Piccini JP. Chocolate and prevention of atrial fibrillation: what is bad for the pancreas might be good for the atria? Heart 2017;103:1141-2.

3 Wilson S, Newby DE. Predicting long-term bleeding risk after acute coronary syndrome: a step closer to optimising dual antiplatelet therapy duration? Heart 2017:103:1143-5.

4 Alfredsson J, Neely B, Neely ML, et al. TRILOGY ACS Investigators. Predicting the risk of bleeding during dual antiplatelet therapy after acute coronary syndromes. Heart 2017;103:1168-76.

5 Ledwoch J, Fuernau G, Desch S, et al. Drugeluting stents versus bare-metal stents in acute myocardial infarction with cardiogenic shock. Heart 2017;103:1177-84

6 Kübler P, Reczuch K. Optimal stent treatment of cardiogenic shock complicating acute myocardial infarction: bare-metal or drug-eluting stent? Heart 2017; 103:1146-7.

7 Rajani R, Modi B, Ntalas I, et al. Non-invasive Fractional Flow Reserve using Computed Tomographic angiography: where are we now and where are we going? Heart 2017;103:1216-22.

8 Chen T, Jones TL, Krieger EV. 21 -year-old with exertional dyspnoea. Heart 2017;103:1215. 